\title{
Catalysis as it goes
}

\author{
In situ studies are very important to advance our comprehension of catalytic reactions and are expected to be \\ boosted by the development of more powerful analytical tools.
}

con haracterizing a catalytic process is a complex and yet crucial task. In fact, the information generated has a large impact on the practicality of a given process and provides insights that can lead to further optimization of any corresponding industrial application. On one side, changes in the composition of reagents and products have to be followed over time in order to define the kinetic parameters of the process; on the other, the evolution of the catalyst's structure must be followed to gain, for example, insights into the possible deactivation paths. Although the reactants and catalyst can be studied independently, it is their interplay that determines the overall outcome of a reaction. That is why we study them when they are together: in situ.

The need to study a catalyst while the reaction is happening, in order to capture transient states existing only in the presence of reagents, was recognized since the early days of modern catalysis research. Michel Boudart, for example, while discussing the concept of 'induced heterogeneity' on catalytic surfaces, wrote in 1952: "A consequence of the dynamic picture of a catalytic surface presented here is the necessity of devising methods for characterizing the surface during the global catalytic reaction" ${ }^{1}$. The technology of that time rarely allowed for studies of that kind. However, only a few years later, the adsorption of gaseous reactants on the surface of a catalyst using infrared spectroscopy - in this case, $\mathrm{NH}_{3}$ on the surface of a cracking catalyst - was measured $^{2}$, as a prelude to the field of in situ characterization in catalysis.

Sixty years later, the existence of differences between a catalyst out of its working environment and a catalyst in action is largely supported by experimental data. The field of in situ characterization has become an established area of research, which in many cases has advanced our understanding of catalytic reactions and allowed their optimization and improvement.

However, as for many other vibrant areas of chemistry, the field is still characterized by several limitations and challenges. And in many cases technical limitations impose such limits. In situ techniques can be roughly divided into methods in which photons interact with the analyte and methods based on electrons as the information carrier ${ }^{3}$. Nuclear magnetic resonance techniques as well as vibrational spectroscopies, which belong to the first group, can be nowadays applied during catalysis to follow the changes associated with the reactants under realistic reaction conditions, for both homogenous and heterogeneous processes. In many cases, for instance, harsher reaction conditions do not represent a real obstacle. In the case of electron-based techniques, however, operational constraints imposed by the instruments have limited the possibilities to analyse a catalyst under realistic reaction conditions, especially in the presence of high-pressure gases or solvents. Take as examples X-ray photoelectron spectroscopy (XPS) or microscopy techniques that are usually employed to image the surface of heterogeneous catalysts: due to the limited range of pressures that can be currently applied in, for instance, transmission electron microscopy (TEM) systems, it is still difficult to perform microscopy for a reaction that requires high pressure.

Such limitations are echoed by terms such as quasi in-situ spectroscopy, which are often encountered in the catalysis literature to describe those studies that can only provide information under approximate reaction conditions. Possibly, the same need for clarity inspired Bañares to coin the term operando, in order to emphasize an analysis that "involves the simultaneous use of an in situ spectroscopy during catalytic operation and on-line activity measurement"4.

\section{While reactants and catalysts can be studied independently, it is their interplay that deter- mines the overall outcome of a reaction. That is why we study them when they are together: in situ.}

Certainly, terminology takes its own time to get established, and while the concepts may be well defined in the literature, the words in situ and operando are occasionally employed as synonymous by catalysis researchers, as observed by editors at ACS Catalysis ${ }^{5}$. We recently tried to capture a snap-shot of the perception that the community has of the terms in situ and operando, with an informal Twitter poll. Interestingly, when asked if it is always possible in practice to distinguish between the two, $47 \%$ of the participants considered some cases as grey areas, whereas for $36 \%$ of the voters the differences remain unclear. Conversely, only $17 \%$ considered the two cases as always clear to distinguish. Obviously, based on sample size and sample selection, this poll lacks statistical significance, but it suggests that attention should be given to specifying the context and implications of the terms in situ and operando to better avoid confusion.

Beyond terminology issues, however, we are confidently looking forward to the continuing rapid development of the field of in situ catalysis studies. In this regard, it is worth recalling that each analytical technique can only provide specific and partial information, while the full understanding of a catalytic process may require inputs from different methodologies at the same time. Here, technical advances in the design and engineering of integrated analytical instruments is expected to make the difference in the coming years.

Promising examples in this direction come from the development of integrated platforms such as 'The TUBE. This facility, of which installation at the University of Lorraine in Nancy was completed in 2017, provides access to multiple techniques for material synthesis and characterization, which are all interconnected under ultra-high vacuum by a 40-metre-long pipe. Samples can be moved between different preparation and analysis stations within a consistent environment. An additional 30-metre-long tube dedicated to industrial research and technology transfer ' is further integrated into the platform (http://go.nature. com/2F6Qmbw). A similar effort is being pursued at the Suzhou Institute of Nano-Tech and Nano-Bionics, in China. In this case, the Vacuum Interconnected Nanotech Workstation (NANO-X, http://english.sinano.cas.cn/au/NANOX/) 
features material growth equipment interconnected by ultra-high vacuum pipes to an array of characterization systems, which include TEM and scanning-electron microscopy (SEM) tools, as well as angleresolved photoemission spectroscopy (ARPES) and XPS tools, among others.

The primary aim of such facilities is the synthesis and characterization of materials under controlled conditions (vacuum) to avoid the risk of contaminations, and thus they may not always provide realistic reaction environments suitable for in situ studies. However, such designs may inspire the development of facilities specifically dedicated to the in situ analysis of catalytic processes, allowing for multiple analysis techniques to be performed on single systems under realistic conditions. This technological innovation and investment could drive our understanding of catalysis as it goes.
Published online: 8 March 2018

https://doi.org/10.1038/s41929-018-0050-4

References

1. Boudart, M. J. Am. Chem. Soc. 74, 1531-1535 (1952).

2. Mapes, J. E. \& Eischens, R. P. J. Phys. Chem. 58 1059-1062 (1954).

3. Dou, J., Sun, Z., Opalade, A. A., Wang, N., Fu, W. \& Tao, F. F. Chem. Soc. Rev. 46, 2001-2027 (2017).

4. Bañares, M. A., Guerrero-Pérez, M. O., Fierro, J. L. G. \& Garcia Cortez, G. J. Mater. Chem. 12, 3337-3342 (2002).

5. Jones, C. W., Tao, F. F. \& Garland, M. V. ACS Catal 2, 2444-2445 (2012). 